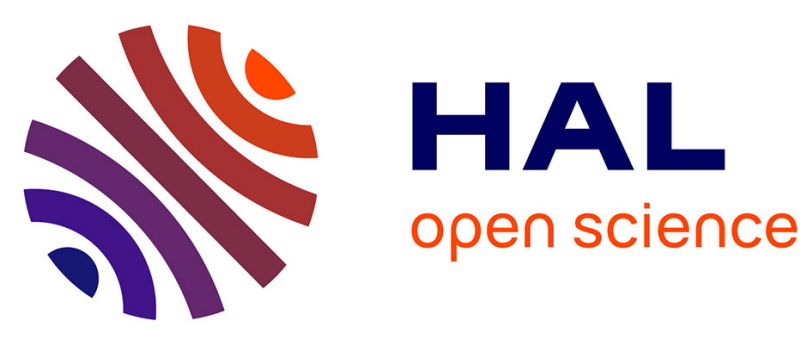

\title{
Harmonic Magnification by Time Reversal based on a Hammerstein Decomposition
}

\author{
Sébastien Ménigot, Jean-Marc Girault
}

\section{To cite this version:}

Sébastien Ménigot, Jean-Marc Girault. Harmonic Magnification by Time Reversal based on a Hammerstein Decomposition. IEEE International Ultrasonic Symposium 2016, IEEE UFFC, Sep 2016, Tours, France. 10.1109/ULTSYM.2016.7728742 . hal-01371264

\section{HAL Id: hal-01371264 \\ https://hal.science/hal-01371264}

Submitted on 24 Nov 2016

HAL is a multi-disciplinary open access archive for the deposit and dissemination of scientific research documents, whether they are published or not. The documents may come from teaching and research institutions in France or abroad, or from public or private research centers.
L'archive ouverte pluridisciplinaire HAL, est destinée au dépôt et à la diffusion de documents scientifiques de niveau recherche, publiés ou non, émanant des établissements d'enseignement et de recherche français ou étrangers, des laboratoires publics ou privés. 


\title{
Harmonic Magnification by Time Reversal based on a Hammerstein Decomposition
}

\author{
Sébastien Ménigot* ${ }^{* \dagger}$ and Jean-Marc Girault* \\ *Université François-Rabelais de Tours, Inserm, Imagerie et Cerveau UMR U930, Tours, France \\ †sebastien.menigot@univ-tours.fr, corresponding author \\ łjean-marc.girault@univ-tours.fr
}

\begin{abstract}
In tissue harmonic imaging, the contrast has been increased by using nonlinearities. However, enhancing the contrast requires a good harmonic level. Time reversal process is well-known to enhance the backscattered signal by optimizing the transmitted wave. However, it is not well-adjusted for second harmonics, since the nonlinearities of the time reversed harmonic signal will be double the frequency and will be removed by the transducer. To take into account the harmonics, one way consists in modelling the ultrasound system by parallel subsystems with a Hammerstein model. Therefore finding the optimal wave for harmonic generation means to create a matched filter for the subsystem describing the nonlinearity. As described by the time reversal, the first wave propagates to the medium. The backscattered second harmonic components are extracted from a Hammerstein model. The second harmonic signal is time reversed, frequency shifted by a demodulation and sent in the medium. In this study, the nonlinear components could be increased by $15 \mathrm{~dB}$ in comparison to the time reversal process and conventional imaging. Moreover, the SNR can be increased by $7 \mathrm{~dB}$. The second harmonic time reversed waves focus better on the inclusion and wave coherence is preserved. From this point of view, the optimization process can be viewed as an extension of the matched filtering feature of the time reversal principle to second harmonics. ${ }^{1}$
\end{abstract}

\section{INTRODUCTION}

The medical ultrasound imaging systems have been improved by taking into account the nonlinear wave propagation. When ultrasound sinus waves at frequency $f_{0}$ propagate nonlinearly, the echoes received are composed of harmonic components $\left(2 f_{0}\right.$, $\left.3 f_{0}, \ldots\right)$. By extracting each harmonic component, it is possible to obtain ultrasound images with high contrast [1]. Given this success, tissue harmonic imaging has become the native imaging modality in conventional ultrasound scanners.

Since the backscattered nonlinearities are a function of the transmitted signal, enhancing the image quality in harmonic imaging means to find the best transmitted wave. Waveform design can be decomposed in two ways: optimizing the wave in space or optimizing the wave in time. In the first way, beamforming has improved the contrast and the resolution [2], even if many studies focused on a simple transmitter and a synthetic beamforming on reception [3]. The second way consists in finding the temporal waveform. Several studies have proposed a solution for nonlinear imaging. An iterative algorithm searches the features of the wave [4] or directly the values of the samples [5].

Moreover, time reversal optimizes the signal-to-noise ratio (SNR) by combining a waveform design in space and in time thanks to a physical matched filter. It makes possible to focus the wave on small inhomogeneities, without information on the medium and even if the medium is aberrating [6]. Its principle consists in sending a first wave at the frequency $f_{0}$ and in retro-propagating the time reversed echoes. Nevertheless, as the wave relives its past propagation, time reversal reduces the harmonic components [7]. This property has been used to reduced the tissue harmonic components in ultrasound contrast imaging [8]. Therefore, this method is not adjusted to maximize the harmonics components from propagation in tissue.

However, other approaches based on the time reversal principle have been proposed, as for instance the method combining time reversal and harmonics [9]. The second harmonics at $2 f_{0}$ is extracted by filtering, even if this filtering do not guarantee that the received harmonics are equal to the extraction, because of the phase. However, this phase difference makes possible that the time reversed second harmonics focus on the small inhomogeneity by a liner propagation at $2 f_{0}$. Therefore, a direct time reversal of second harmonics is not the matched filter which optimizes the SNR for harmonic components, since the propagation at $2 f_{0}$ is linear. To understand it, one way consists in modelling the ultrasound system by simple parallel subsystems. Such model as a Hammerstein system is sufficient to describe the nonlinear behaviour [10], [11]. Thus the system usually can be decomposed by parallel subsystems where the nonlineary is separated of the linearity. For example, a second order model (shown in Fig. 1) can describe the output from the input. The first subsystem is dedicated to linear output by a linear filter $h_{1}$. In the second subsystem, in order to create the second harmonics, a polynomial function is added to a linear filter $h_{2}$. In time reversal, only the first channel is considered. Therefore, after the transmission of the time reversed fundamental component at

${ }^{1}$ DOI : $10.1109 /$ ULTSYM.2016.7728742

(c) 2016 IEEE. Reprinted, with permission, from Sébastien Ménigot and Jean-Marc Girault, Harmonic magnification by time reversal based on a Hammerstein decomposition, 2016 IEEE International Ultrasonics Symposium (IUS), 2016. This material is posted here with permission of the IEEE. Such permission of the IEEE does not in any way imply IEEE endorsement of any of the Université François Rabelais de Tours' products or services. Internal or personal use of this material is permitted. However, permission to reprint/republish this material for advertising or promotional purposes or for creating new collective works for resale or redistribution must be obtained from the IEEE by writing to pubs-permissions@ieee.org. 
$f_{0}$, the SNR of the linear output is optimal thanks to a matched filter of the first channel. However, finding the optimal wave for harmonic generation means to create a matched filter for the second channel and its solution is not trivial. Indeed, after the transmission of the time reversed second harmonics at $2 f_{0}$, on the one hand, the $2 f_{0}$ retropropagated component is due to a linear process (first channel) and so not optimal (since it is different to the time reversal solution). On the other hand, the nonlinearities double the frequency at $4 f_{0}$ because of the square function in the second channel. The matched filter for the second harmonics is thus disrupted by the nonlinear effect (modelled here by a square function).

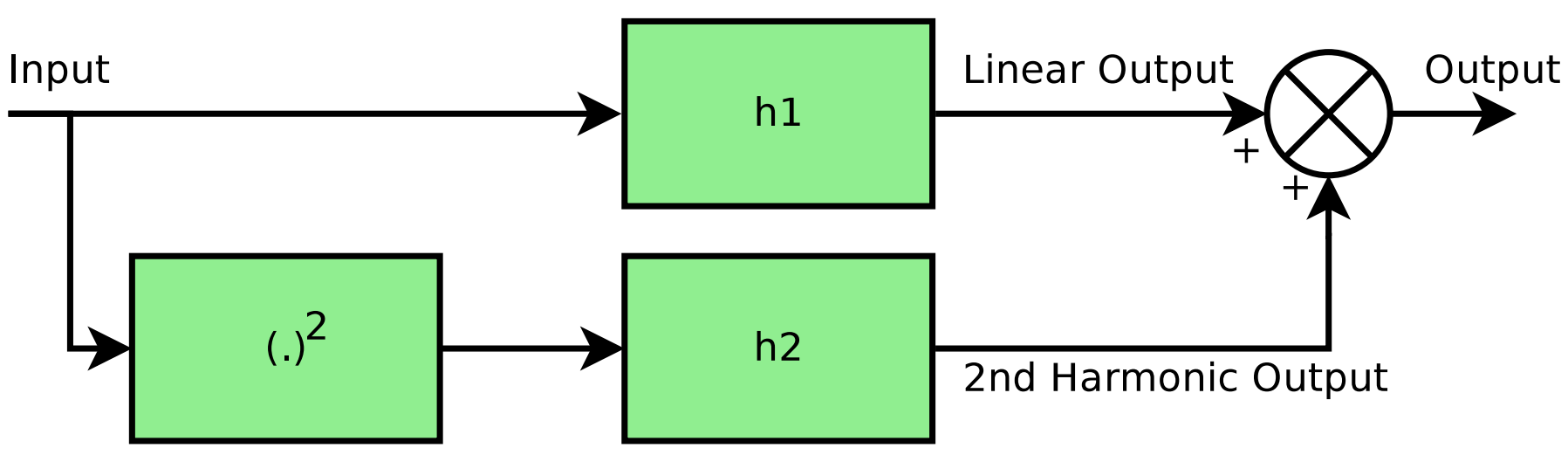

Fig. 1. Block diagram of a second order polynomial Hammerstein model.

Our objectives is thus to find the optimal wave to optimize the harmonic generation by taking into account this frequency doubling and without increasing the transmit power. We proposed to adjust time reversal by adding a new step. By assuming that the ultrasound system can be modelled as a Hammerstein decomposition, the matched filter for harmonics could be obtained by reversing time and by annihilating the harmonic effects. This annihilation could be simply obtained by inversing the function responsible to the nonlinear effect in the Hammerstein model. In this paper, this method was applied in simulation. Moreover, its feasability was just evaluated in order to magnify the second harmonics only.

\section{HARMONIC MAGNIFICATION}

As previously explained, the harmonic magnification is based on the time reversal principle according to two steps (Fig. 2). The first step (switches in position 1) consists in sending a first standard excitation $x_{\text {standard }}(n)$ and in extracting the second harmonic backscattered components $y_{N L}(n)$ by a Hammerstein model. However, by retro-propagating this second harmonics directly, the components at $2 f_{0}$ are due to a linear effect, since the subsystem describing the harmonic behaviour double the frequency. Thus, in a second step (switches in position 2), it is required to annihilate the nonlinear effects by inverting the nonlinear contribution and then by reversing time. This optimal signal $x_{\text {opt }}(n)$ is finally retro-propagated into the medium.

\section{A. Harmonic extraction}

The harmonic extraction is based on a Hammerstein model. Each model is usually decomposed by parallel subsystems as a polynomial function and a linear filter. However, the polynomial functions are inverse functions only on $\mathbb{R}^{+}$. Thus, to garantee the inversion of subsystems, the polynomial functions are replaced by frequency shifting (Fig. 2) thanks to a modulation. Note that this change is also possible, since the continuous component usually modelled thanks to the square function is here filtered by the transducer. The signal modelling $\hat{y}(n)$ of the basckattered signal $y(n)$ can be thus written as:

$$
\hat{y}(n)=\sum_{p=1}^{P} \sum_{m=1}^{M} h_{p}(m) x_{\text {standard }}(n-m) \cdot C_{p}(n),
$$

where $n$ is the discrete time, $M$ the memory of the Hammerstein model, $P$ its order, $x_{\text {standard }}(n)$ the input model and $C_{p}(n)=\cos \left(2 \pi \frac{(p-1) f_{0} n}{F_{s}}\right)$ with $F_{s}$ the sample frequency. Note that here the order $P$ has to be set 2 in order to extract the second harmonics. Therefore, the Hammerstein modelling is solved to find the linear filter coefficients $h_{p}$.

This problem can be written with an algebraic formula, such as :

$$
\hat{\mathbf{z}}=\mathbf{X}^{T} \mathbf{h},
$$

where $\hat{\mathbf{y}}=[y(M+1), \ldots, y(N)]^{T}$ with $N$ the sample quantity and ${ }^{T}$ the symbol of the vector transposition, $\mathbf{h}$ the vector of coefficients

$$
\mathbf{h}=\left[h_{1}(1), \ldots, h_{1}(M), h_{2}(1), \ldots, h_{2}(M)\right]
$$




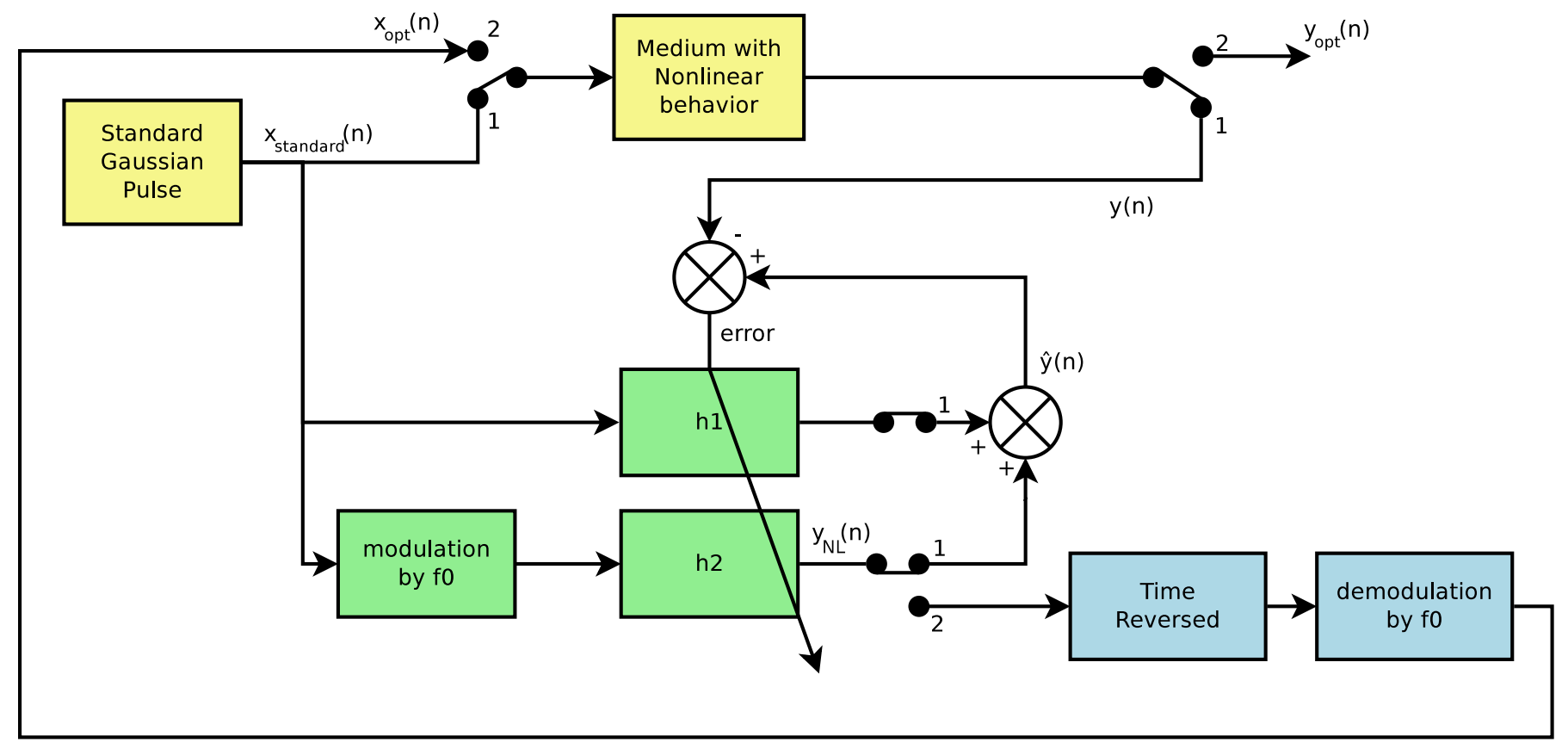

Fig. 2. Block diagram of harmonic magnification by time reversal based on a Hammerstein decomposition. The yellow functions are the standard imaging. By setting the switches in position 1, the second harmonic components can be extracted by the Hammerstein model in green. Finally the second harmonic components are used to optimize the the basckettered harmonics in blue.

and the matrix of input signals $\mathbf{X}=\left[\mathbf{x}_{1} \mathbf{x}_{2}\right]$ with

$$
\mathbf{x}_{p}=\left(\begin{array}{llll}
v_{p}(M) & v_{p}(M+1) & \ldots & v_{p}(N) \\
v_{p}(M+1) & v_{p}(M+2) & & 0 \\
\vdots & & . \cdot & \\
v_{p}(N) & 0 & & 0
\end{array}\right)
$$

and $v_{p}(n)=x(n) C_{p}(n)$.

Finding a solution minimum the error between the signal modelling $\hat{y}(n)$ and the backscattered signal $y(n)$ can be solved with a pseudo-inversion, such as:

$$
\mathbf{h}=\left(\mathbf{X}^{T} \mathbf{X}\right)^{-1} \mathbf{X}^{T} \mathbf{y} .
$$

Finally, the second harmonic extraction is based on the coefficients $\mathbf{h}_{2}=\left[h_{2}(1), \ldots, h_{2}(M)\right]^{T}$. The second harmonic signal $\mathbf{y}_{N L}$ can be modelled as:

$$
\mathbf{y}_{N L}=\mathbf{x}_{2}^{T} \mathbf{h}_{2} .
$$

Note that a Matlab program of a Hammerstein decomposition is available on RunMyCode.org (http://www.runmycode.org/coder/view/239!

\section{B. Time Reversal for Second Harmonics}

After the second harmonic extraction, the second harmonic signal $\mathbf{y}_{N L}$ is time reversed. Moreover, since we assume that the second harmonic can be modelled by a frequency shifting and a linear filter, annihilating the effects of the second harmonics consists in an "opposite" frequency shifting by a demodulation. Moreover, this inverse frequency shifting has to take into account the phase of the backscattered second harmonic signal $\mathbf{y}_{N L}$. Thus the best transmitted signal to magnify the second harmonics can be written as follow:

$$
x_{\text {opt }}(n)=A \cdot y_{N L}(n) \cdot \cos \left(2 \pi f_{0} \frac{n}{F_{s}}+\phi\right),
$$

where $A$ is a coefficient in order to preserve the transmit power equal to the initial transmitted signal $x_{\text {standard }}(n)$. The phase difference $\phi$ is obtained by comparing the phase of the second harmonic signal $\mathbf{y}_{N L}$ and the phase of the cosinus:

$$
\phi=\arctan \left(\frac{\varphi\left(\mathbf{y}_{N L}\right)}{\varphi(\cos )}\right) \text {, }
$$


with $\varphi\left(\mathbf{y}_{N L}\right)$ is the phase of the the second harmonic signal $\mathbf{y}_{N L}$ and the $\varphi(\cos )$ the phase of a cosinus at the frequency $f 0$. Note these phases are numerically obtained by a fast Fourier transform.

\section{Simulation Model}

In order to validate the feasibility, we used realistic simulations which have already been proven in medical ultrasound imaging [4]. The simulation model is composed of different phases: transmission, 2D nonlinear propagation and reception.

At the transmitter, a Gaussian pulse $x_{\text {standard }}(n)$ at a centre frequency $f_{0}$ of $2 \mathrm{MHz}$ and with a bandwidth of $50 \%$ is generated digitally with Matlab (The Mathworks, Natick, MA, USA). It is transmitted to an 8-element probe with a pitch of $400 \mu \mathrm{m}$ and filtered by its transfer function from a realistic transducer, centred at $f_{c}=4 \mathrm{MHz}$ with a fractional bandwidth of $80 \%$ at $-3 \mathrm{~dB}$. Note that the signal $x_{\text {standard }}(n)$ is set in order to guarantee the reception of second harmonics. The pulse wave generated is propagated nonlinearly into an attenuating medium (Fig. 3), i.e. a $2 \mathrm{~mm}$-broad inclusion of a second tissue sample is inserted into the first tissue at $8 \mathrm{~mm}$ below the surface). Note that these small sizes were chosen to reduce the computation time of the propagation simulation. Moreover a small target with a high impedance is inserted at $12 \mathrm{~mm}$. The 2D nonlinear wave propagation is solved using the Anderson model based on a pseudo-spectral derivative and a time-domain integration algorithm [12].

\section{Ultrasound Probe}

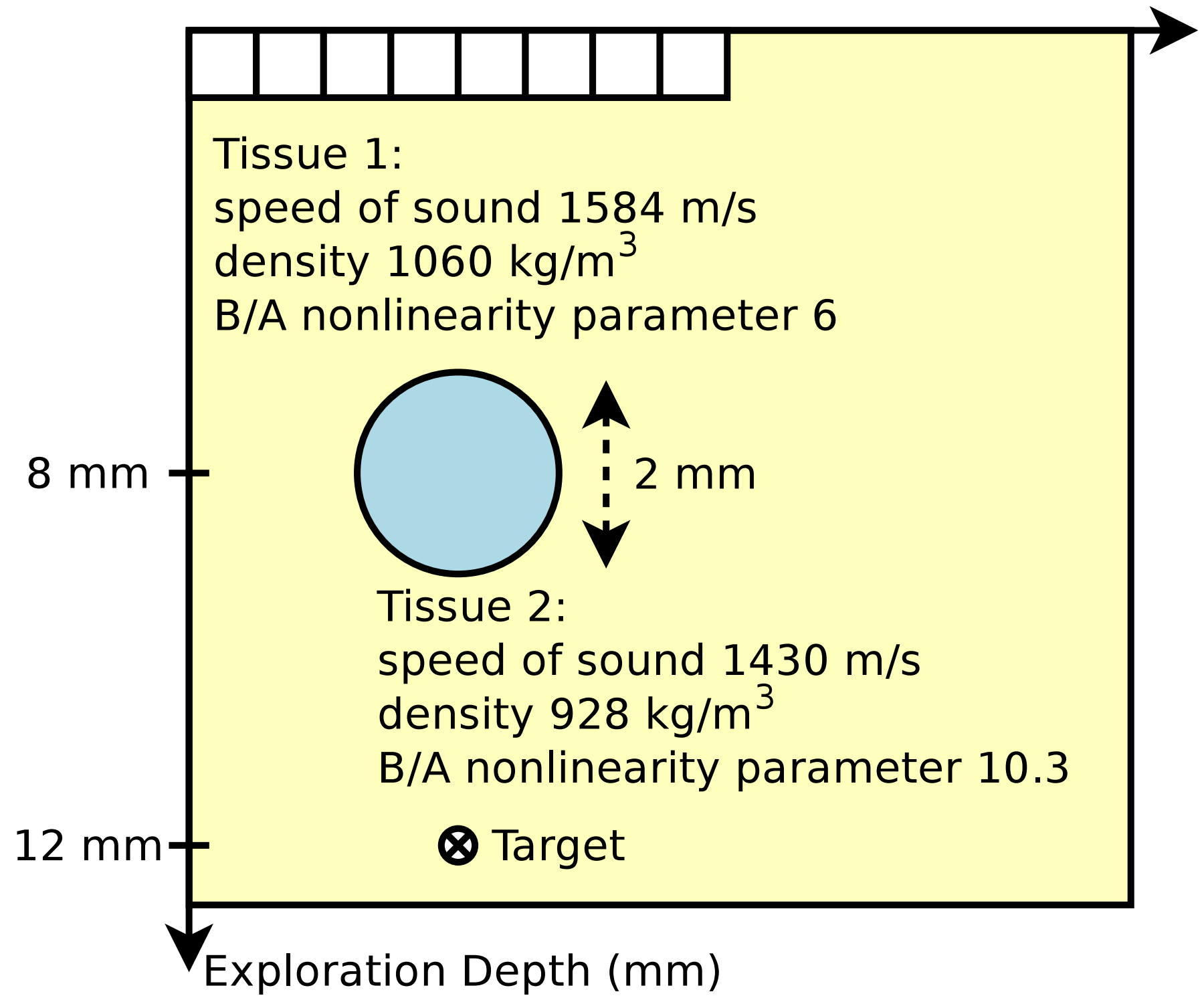

Fig. 3. Grid of medium properties. The ultrasound transducer was at a depth of $0 \mathrm{~mm}$, here at the top. Note that this scheme is not to scale. 
Finally, the signals $y(n)$ basckattered by tissue are recorded and filtered by the transfer function of the transducer. The harmonic magnification process is separately applied for basckattered signal $y(n)$ of each element of the probe. Finally the best signals $x_{o p t}(n)$ to magnify the second harmonics are transmitted to the medium and the backscattered signal $y_{o p t}(n)$ are recorded.

\section{RESUlts}

Fig. 4 shows the input and second harmonic output signals in the case of standard time reversal and in the case of time reversal for second harmonics. Note that the second harmonic extraction was made by a second-order Hammerstein decomposition. Their respective spectra are depicted in Fig. 4a. As an illustration, the spectra of the initial Gaussian pulse at $2 \mathrm{MHz}$ and the the harmonic basckettered signal after focusing are shown. Note that this focusing is obtained by a standard beamforming using Euclidean geometry [2].

By using time reversal, the second harmonic waves focused on the target position. However, if the time reversal was dedicated to the second harmonics, the amplitude of the second harmonic signal was higher. This results was confirmed on the spectra with a second harmonic components higher than $15 \mathrm{~dB}$ by using time reversal for second harmonics than standard time reversal or than standard beamforming.
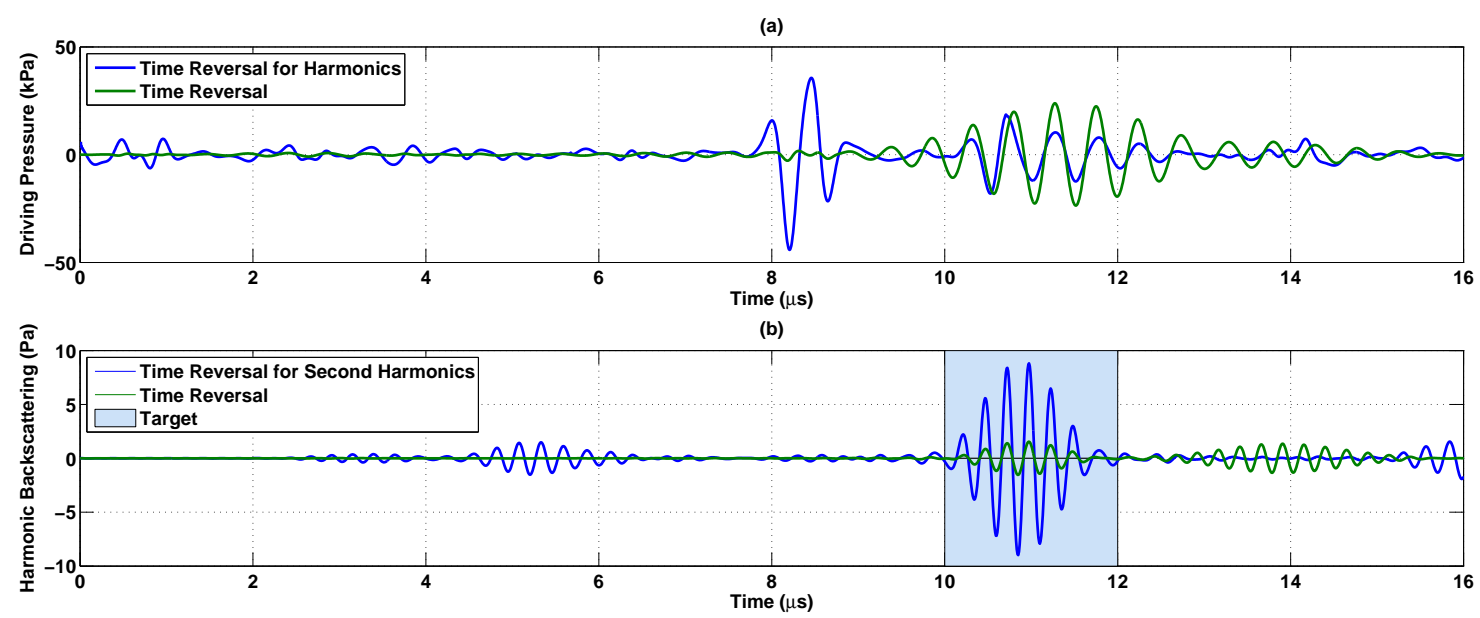

(c)

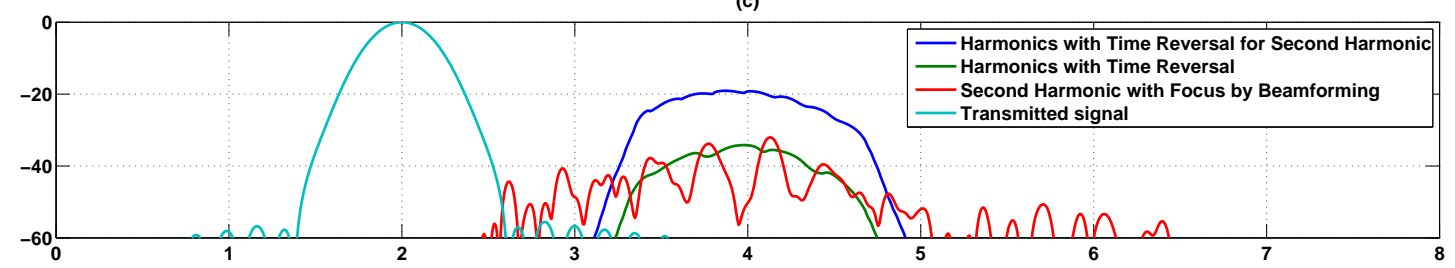

Fig. 4. (a) Driving pressures transmitted to the medium thanks to the center element of the probe in case of time reversal and of time reversal for second harmonics. (b) Harmonic backscattered signals received by the center element of the probe in case of time reversal and and of time reversal for second harmonics. (c) Spectra of Harmonic backscattered signals in case of time reversal and and of time reversal for second harmonics. As an illustration, the spectra of the initial Gaussian pulse and the harmonic basckettered signal after focusing by beamforming are depicted.

Moreover, on the harmonic backattered signals, the SNR was measured in case of time reversal for second harmonics using frequency shifting. This SNR is compared to the method using time reversed second harmonics without frequency shifting as in [9], standard time reversal and a focusing by beamforming. They are shown in Fig. 5. The SNR could increase sighlty by using time reversal rather a standard beamforming. However, the SNR increase could reach $7 \mathrm{~dB}$ in comparison the time reversal for second harmonics. Note that no harmonic extraction were used to evaluated the SNR on time reversed second harmonics without frequency shifting, since the retropropagation was a linear process.

Finally, as an illustration, pressure propagating in the medium by using time reversal for second harmonics are shown at the beginning of the propagation and at the focusing. As soon as the beginning of the propagation, the wavefront was deformed to take into account the inclusion. Thus the wave focused even with the presence of this inclusion. 


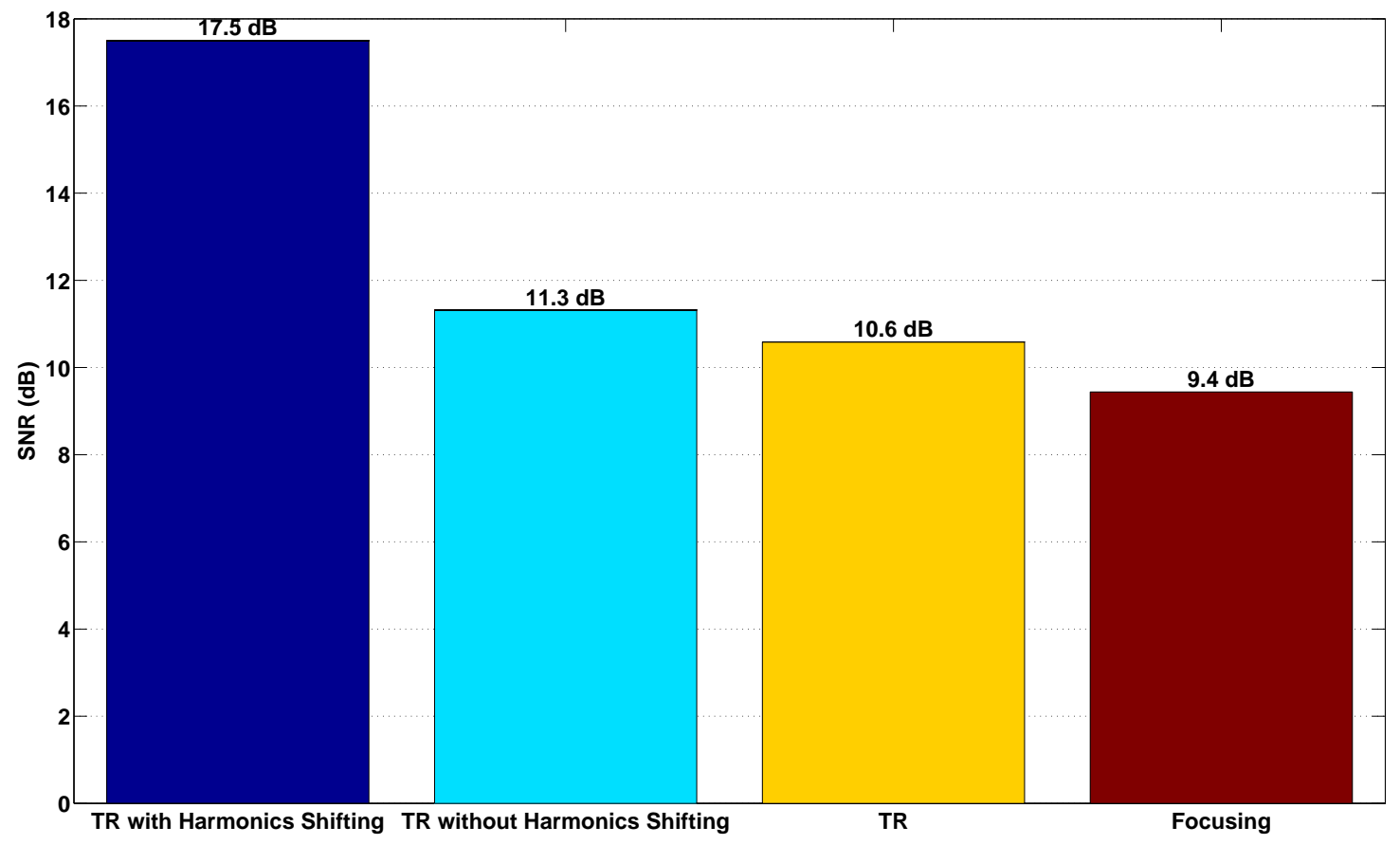

Fig. 5. SNR measured on harmonic backscattered signals obtained with time reversal (TR) for second harmonics using frequency shifting, without frequency shifting, standard time reversal and a focusing by beamforming.

(a)

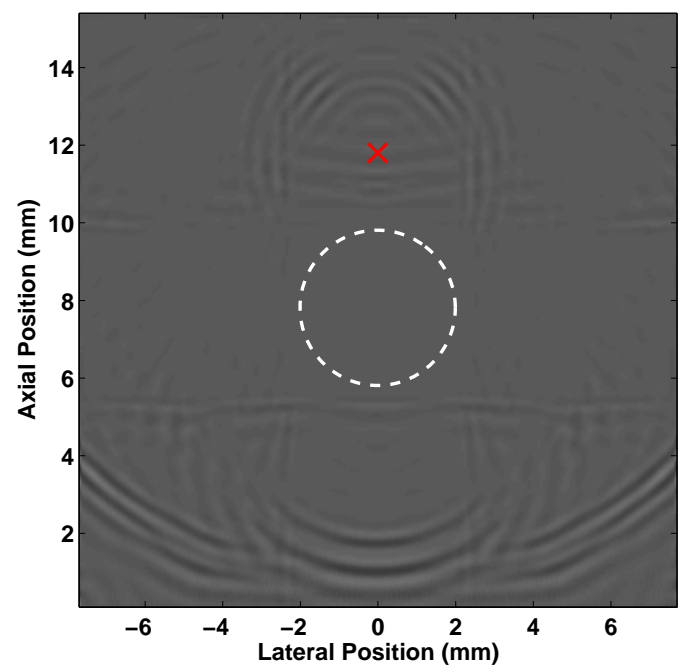

(b)

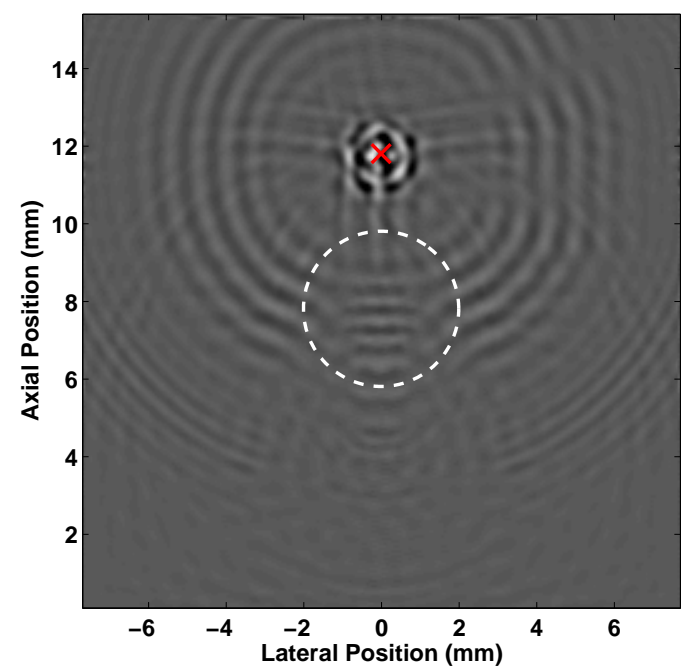

Fig. 6. Image of pressure propagating in the medium by using time reversal for second harmonics at the beginning of the propagation (a) and at the focusing (b). As an illustration, the target is depicted with a red cross and the inclusion with a white line. Note that the ultrasound probe was located at 0 mm (on the bottom in the figures).

\section{DisCUSSIONS AND CONCLUSION}

In contrast with the standard time reversal, the method presented here is adjusted to magnify the second harmonics. By finding a wave which could take into account the medium complexity, the wave focused on the target and wave coherence is preserved. Therefore it generated a higher quantity of second harmonics and it made possible to highly increase the SNR. 
Moreover, this magnification lies in the annihilation of the harmonic effects before sending the time-reversed signal. This annihilation was possible because we assumed that the ultrasound system could be modelled by a Hammerstein model using frequency shifting. However, this assumptions could become wrong for high nonlinearity. Moreover, taking into account the phase was crucial for the demodulation. However, the phase estimation was not precise and yet was sufficient to improve the SNR.

From this point of view, the optimization process can be viewed as an extension of the matched filtering feature of the time reversal principle to second harmonics. This method could help to improve the harmonic imaging in the case where the aberration phase can be high. We will carry on validating this concept experimentally. Moreover, the method will be generalized at high order of nonlinearity by adjusting the nonlinear annihilation.

\section{REFERENCES}

[1] P. N. Burns, "Instrumentation for contrast echocardiography," Echocardiography-A Journal Of Cardiovascular Ultrasound And Allied Techniques, vol. 19, no. 3, pp. 241-258, Apr. 2002.

[2] T. Szabo, Diagnostic Ultrasound Imaging: Inside Out. London, UK: Academic Press, 2004.

[3] J. A. Jensen, S. I. Nikolov, K. L. Gammelmark, and M. H. Pedersen, "Synthetic aperture ultrasound imaging," Ultrasonics, vol. 44, Supplement, no. 0, pp. e5-e15, 2006.

[4] S. Ménigot, J.-M. Girault, I. Voicu, and A. Novell, "Optimization of contrast to tissue ratio by frequency adaptation in pulse inversion imaging," IEEE Transactions on Ultrasonics, Ferroelectrics, and Frequency Control, vol. 59, no. 11, pp. 2431-2438, Nov. 2012.

[5] S. Ménigot and J.-M. Girault, "Optimization of contrast resolution by genetic algorithm in ultrasound tissue harmonic imaging," Ultrasonics, no. 71, pp. 231-244, Sep. 2016.

[6] M. Fink, "Time-reversal of ultrasonic fields .1. basic principles," IEEE Transactions on Ultrasonics Ferroelectrics and Frequency Control, vol. 39, no. 5, pp. 555-566, Sep. 1992.

[7] M. Tanter, J.-L. Thomas, F. Coulouvrat, and M. Fink, "Breaking of time reversal invariance in nonlinear acoustics," Physical Review E, vol. 64, no. 1, Jun. 2001

[8] O. Couture, J. F. Aubry, G. Montaldo, M. Tanter, and M. Fink, "Suppression of tissue harmonics for pulse-inversion contrast imaging using time reversal," Physics in medicine and biology, vol. 53, no. 19, pp. 5469-5480, Oct. 2008.

[9] T. Goursolle, S. Dos Santos, O. Bou Matar, and S. Calle, "Non-linear based time reversal acoustic applied to crack detection: Simulations and experiments," International Journal of Non-Linear Mechanics, vol. 43, no. 3, pp. 170-177, 2008.

[10] K. Narendra and P. Gallman, "An iterative method for the identification of nonlinear systems using a hammerstein model," IEEE Transactions on Automatic control, vol. 11, no. 3, pp. 546-550, 1966.

[11] F. Sbeity, S. Ménigot, J. Charara, and J.-M. Girault, "Contrast improvement in sub- and ultraharmonic ultrasound contrast imaging by combining several hammerstein models," International Journal of Biomedical Imaging, vol. 2013, p. 9, Sep. 2013.

[12] M. E. Anderson, "A 2d nonlinear wave propagation solver written in open-source matlab code," in Proceeding IEEE Ultrasonic Symposium, San Juan, Puerto Rico, Oct. 2000, pp. 1351-1354. 